\title{
Aging Time on Rutile Pigment Production via Short Sulfate Process
}

\author{
Cong-Xue $\operatorname{TIAN}^{1,2}$ a \\ ${ }^{1}$ Panzhihua University, Panzhihua 617000, China \\ 2 State Key Laboratory for Vanadium \& Titanium Testing, Panzhihua 617000, China \\ aemail: tcx7311@163.com
}

Keywords: Aging time, F value, rutile, short sulfate process, pigment

\begin{abstract}
Rutile $\mathrm{TiO}_{2}$ white pigment was produced by using low concentration industrial $\mathrm{TiOSO}_{4}$ solution as raw material via short sulfate process through self-generated seeded thermal hydrolysis route. The aging time and $\mathrm{F}$ value have significantly influenced the structure and pigment properties of rutile $\mathrm{TiO}_{2}$ white pigment. The samples were characterized by XRD, particle size distribution and pigment properties test. Appropriate aging time and $\mathrm{F}$ value were beneficial to promote hydrolysis process in a proper way and obtaining favorable structure and high quality white pigment. The optimized aging time was of $25 \mathrm{~min}, \mathrm{~F}$ value of 1.90.
\end{abstract}

\section{Introduction}

Titanium dioxide $\left(\mathrm{TiO}_{2}\right)$ has the properties of higher opacity, smaller crystal size, and inertness, and it is the best choice of white pigment for its higher refractive index. It has been used in many fields such as paints, paper, fibers, cosmetics, and so on. The production of titanium dioxide pigments via the hydrolysis precipitation of titanyl sulfate, more commonly known as the sulfate process, is one of the most important methods worldwide, especially in China ${ }^{[1]}$. There is abundant of ilmenite in Panzhihua, only the Sulfate Process can be adopted to produce white pigment due to its high content of calcium and magnesium. Titanium dioxide in the form of rutile is usually precipitated from hot concentrated titanyl sulfate solution via seeds inoculating or self-seeded process. And the hydrolysis of $\mathrm{TiOSO}_{4}$ solution is the core and determines the final pigment quality and yield. The particle morphology, particle size and its distribution of the hydrolyzed product (namely the hydrated titanium dioxide or metatitanic acid), determines the structure and quality of titania at some extent ${ }^{[2]}$. Though the precipitation step has been used in industry since 1918, there is no try in using low concentration $\mathrm{TiOSO}_{4}$ solution (namely unconcentrated) to produce $\mathrm{TiO}_{2}$ white pigment. In the self-seeded generated precipitation, the formation of $\mathrm{TiO}_{2}$ occurs through a series of steps involving ionic reactions to form precursors for polymerisation, during which olation and oxolation gives birth to nuclei, which continue to grow and aggregate to the final form ${ }^{[3]}$. And the influences of solution composition and acidity on the rate of precipitation and yield have been investigated widely ${ }^{[46]}$, all the reports showed that the hydrolysis process went through three steps, i.e. grain formation of crystallization, nuclei growth and hydrated titania precipitation, hydrated titania aggregation and composition changes of the precipitation. Homogeneous nucleation and growth of mono-dispersed spherocrystals of sulfur and hydrated titanium dioxide of anatase modification were studied by a new coherent optical method for measurement of the relative scattering coefficient based on alternative use of laser radiation of various wavelengths ${ }^{[7]}$. Sathyamoorthy investigated the particle formation process of anatase and the mechanisms behind particle formation during anatase precipitation from seeded concentrated titanyl sulfate solution ${ }^{[8]}$. All these reports focused on the particle size and its distribution, as well as the structure of the crystal and precipitation, but with no further connection with its pigment properties and no detail information of the precipitation. The particle size and distribution, crystal structure, even the precipitation process was the heart of $\mathrm{TiO}_{2}$ production and determines the final pigment quality and yield. Our previous work had investigated some hydrolysis factors influence on the structure and pigment properties of $\mathrm{TiO}_{2}$ via self-generated seed process by using concentrated $\mathrm{TiOSO}_{4}$ solution as raw material ${ }^{[9 \sim 10]}$. 
Using the low concentration $\mathrm{TiOSO}_{4}$ solution as raw material to produce $\mathrm{TiO}_{2}$ white pigment will lower the cost, reduce energy consumption and shorten the process. But there are few reports on $\mathrm{TiO}_{2}$ white pigment production through low concentration titanyl sulfate, because the decrease of titanium concentration, acidity and hydrolysis temperature leads to the change of hydrolysis rate, particle growth and aggregation, different from traditional sulfate process, resulting in difference in structure and pigment properties. It is of great importance to discuss the effects of hydrolysis parameters via low concentration $\mathrm{TiOSO}_{4}$ solution on the structure and pigment performances. Herein we reported using the low concentration $\mathrm{TiOSO}_{4}$ solution as raw material, obtained by acidolysis of titanium slag and ilmenite, then leaching, purifying and without concentration, $\mathrm{TiO}_{2}$ white pigment was prepared by hydrolyzing, washing, doping and calcinations via self-seeded route. The aging timeduring hydrolysis and $\mathrm{F}$ value for the low concentration $\mathrm{TiOSO}_{4}$ solution was investigated in order to determine the optimized synthesis conditions.

\section{Experimental}

Low concentration $\mathrm{TiOSO}_{4}$ solution was taken from the sulfate process (Titanium Industry Factory in Panzhihua, China), and its composition was $\mathrm{TiO}_{2}$ of $184.30 \mathrm{~g} / \mathrm{L}, \mathrm{F}=$ (effective $\left.\mathrm{H}_{2} \mathrm{SO}_{4}\right) / \mathrm{TiO}_{2}=1.85, \mathrm{Fe} / \mathrm{TiO}_{2}=0.29(\mathrm{wt} \%), \mathrm{Ti}^{3+} 2.15 \mathrm{~g} / \mathrm{L}$.

Low concentration $\mathrm{TiOSO}_{4}$ solution and pre-adding water was separately preheated up to $96 \pm 1{ }^{\circ} \mathrm{C}$, and then the $\mathrm{TiOSO}_{4}$ solution was dropped into the pre-adding water in a four mouth flask with refluxing and stirring at constant speed in about $18 \mathrm{~min}$, with volume ratio of $0.16: 1$. After feeding off, the mixture was heated to the boiling point (also called the first boiling point). When the hydrolysis mixture turned into grey color after slight boiling for a few minutes, heating and stirring was immediately stopped to age. After aging for some time, heating and stirring was turned on, and the mixture was then heated to boil again (as called the second boiling point) for another few minutes. After 90min from the second boiling point, 3\% volume distilled water was added in order to increase the hydrolysis rate and keep the slurry concentration at some preferable degree. The hydrolysis process was completed after $2.5 \mathrm{~h}$ from the second boiling point, then the slurry was filtered and washed with diluted sulfate solution and distilled water, then metatitanic acid was obtained. The as-prepared metatitanic acid was doped with calcined seed, zinc salt, potassium salt and phosphate salt, then dried and calcined according to the optimized salt treatment and calcinations conditions. And the rutile $\mathrm{TiO}_{2}$ white pigment was prepared.

The crystal grain size was obtained on X-ray diffractometer (Bruker Advanced D8). Particle size distribution test was carried out on a Malvern particle size analyzer instrument (Zetasizer Nano ZS90). The pigment properties (achromic ability and brightness) were also conducted on the integral ball spectrophotometer (USA, X-Rite Corporation), contrast to the standard sample R930.

\section{Results and Discussion}

The different aging time was conducted for $5 \mathrm{~min}, 15 \mathrm{~min}, 25 \mathrm{~min}, 35 \mathrm{~min}, 45 \mathrm{~min}$. The obtained metatitanic acid samples at different aging time were marked as PTS1, PTS2, PTS3, PTS4, PTS5, and the obtained rutile titania samples were denoted as R1, R2, R3, R4, R5, respectively. The XRD patterns of the metatitanic acid were showed in Fig.1, and the calcined rutile $\mathrm{TiO}_{2}$ sample (sample R3) was showed in Fig.2. 


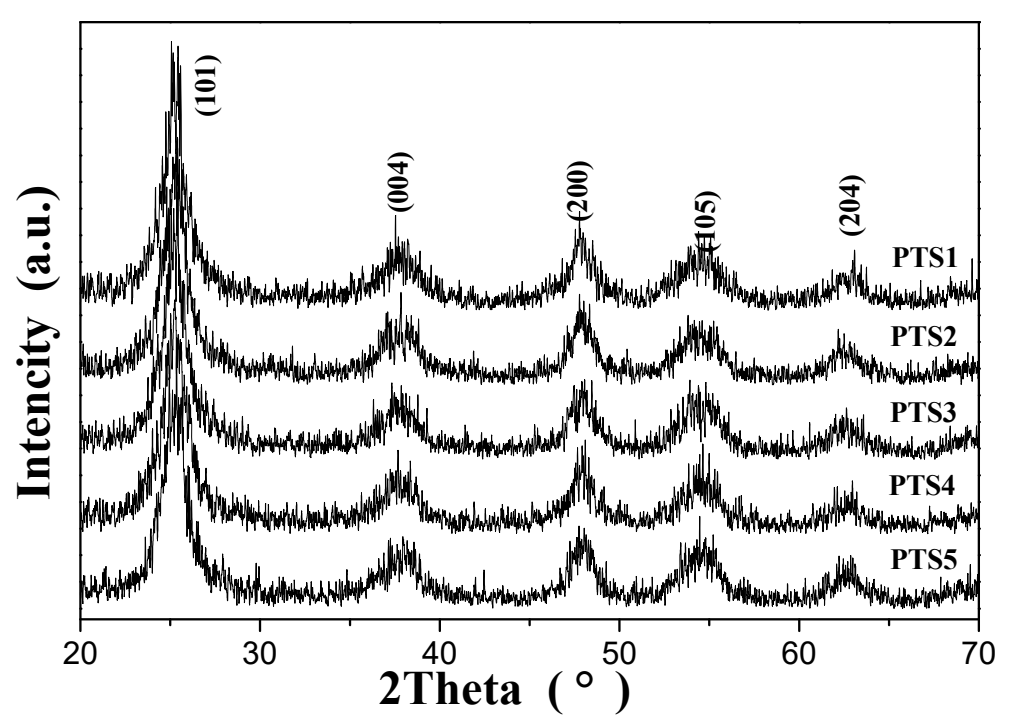

Fig.1 XRD patterns for $\mathrm{H}_{2} \mathrm{TiO}_{3}$ obtained for different aging time

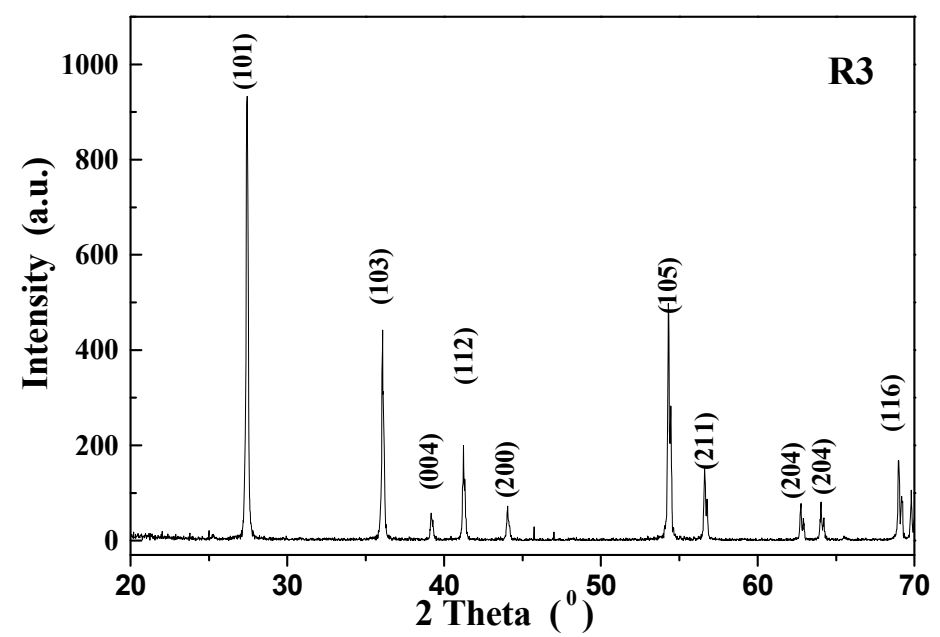

Fig. 2 XRD pattern for the rutile $\mathrm{TiO}_{2}(\mathrm{R} 3)$

All the XRD patterns of metatitanic acid showed clearly anatase phase, consistent with the standard anatase phase pattern (JCPDS 21-1272). As the aging time increased, diffraction peaks became narrower and sharper, suggesting its higher crystallization degree. Its grain size calculated from XRD was gradually decreased from $7.14 \mathrm{~nm}$ to $9.03 \mathrm{~nm}$, in accord with the XRD patterns. The calcined sample R3 showed the entirely rutile phase with high crystallization (showed in Fig.2), with grain size of $103.2 \mathrm{~nm}$. Appropriate crystalline and nice crystal structure was beneficial to improve the pigment properties.

Table 1 the structure and pigment properties of $\mathrm{TiO}_{2}$ obtained for different aging time

\begin{tabular}{ccccccccc}
\hline sample & $\begin{array}{c}\text { Aging } \\
\text { time } \\
(\mathrm{min})\end{array}$ & $\begin{array}{c}\mathrm{Grain}^{2} \mathrm{Hize} \text { of } \\
\mathrm{H}_{2} \mathrm{TiO}_{3}(\mathrm{~nm})\end{array}$ & $\begin{array}{c}D_{A V} \\
(\mathrm{~nm})\end{array}$ & Pdi & \multicolumn{3}{c}{ Achromatic ability } & \multicolumn{2}{c}{ Brightness } \\
$\mathrm{R} 1$ & 5 & 7.14 & 335 & 0.329 & 1600 & 2.32 & 94.18 & 99.64 \\
$\mathrm{R} 2$ & 15 & 7.82 & 316 & 0.274 & 1690 & 2.93 & 94.56 & 100.04 \\
$\mathrm{R} 3$ & 25 & 8.07 & 297 & 0.103 & 1800 & 3.29 & 95.07 & 100.58 \\
$\mathrm{R} 4$ & 35 & 8.65 & 308 & 0.147 & 1710 & 2.85 & 95.01 & 100.52 \\
$\mathrm{R} 5$ & 45 & 9.03 & 321 & 0.245 & 1620 & 2.06 & 94.55 & 100.03 \\
R930 & $/$ & $/$ & $\mathbf{2 8 8}$ & $\mathbf{0 . 1 6 7}$ & $\mathbf{1 9 1 0}$ & $\mathbf{3 . 1 2}$ & $\mathbf{9 4 . 5 2}$ & $/$ \\
\hline
\end{tabular}

The average particle size $\left(D_{A V}\right)$, particle polydispersity (Pdi) and pigment properties of the as-prepared rutile titania white were listed in Table 1 . The average particle size of $\mathrm{TiO}_{2}$ was firstly 
decreased as aging time prolonging, then increased, among them sample R3 was with the smallest $P d i$ value of 0.103, and its average particle size of $297 \mathrm{~nm}$, indicating the narrowest particle size distribution. Appropriate particle size and narrow particle size distribution would also improve its pigment properties. As the aging time increasing, the reducing power (TCS) of the samples firstly increased and then decreased, and the TCS of sample R3 was the highest. The blue phase index $(S C X)$ firstly increased and then decreased as aging time increasing from $5 \mathrm{~min}$ to $45 \mathrm{~min}$, the biggest one was R3 with the value of 3.29. The brightness also had similar variation trend, and aging time of $25 \mathrm{~min}$ was the best, with relative whiteness of $100.58 \%$ as to R930.

As the aging time increasing, the average particle size of $\mathrm{TiO}_{2}$ gradually decreased from $335 \mathrm{~nm}$ to $297 \mathrm{~nm}$, then increased to $321 \mathrm{~nm}$. And its particle polydispersity firstly decreased to the value of 0.103 , then increased to 0.245 , probably due to the number of hydrolysis nuclei. The aim of aging was mainly to control the hydrolysis rate and number of nuclei. When the hydrolysis solution turned into grey color, the critical point of the hydrolysis process with the largest velocity, a large number of nuclei formed to accelerate hydrolysis. To obtain proper fine particle and narrow particle size distribution, aging must be taken to decrease hydrolysis rate to lower the number of new formed nuclei. Shorter aging time was inclined to form larger number nuclei, while longer aging time was on the contrary. Appropriate number of nuclei was beneficial to form proper size particle with narrow particle size distribution, resulting in improving its pigment properties. The optimized aging time was of $25 \mathrm{~min}$. And this was also consistent with Sathyamoorthy's report ${ }^{[1]}$.

The SEM photograph of sample R3 was showed in Fig.3. The rutile titanium dioxide particles showed clear grain outline and with particle size distribution arranging from $70 \mathrm{~nm}$ to $360 \mathrm{~nm}$, and the smaller particle might congregate together to form the larger one, this was consist with average particle size of $297 \mathrm{~nm}$ listed in table 1 . When $\mathrm{TiOSO}_{4}$ solution was initially added to the pre-heated water, it hydrolyzed and formed the white colloid particles, these colloid particles were nuclei which would induce the hydrolysis of $\mathrm{TiOSO}_{4}$ in the desired way, with more pre-adding water volume ratio, forming more nuclei. These nuclei particles were then dispersed and the solution turned into clear with adding more $\mathrm{TiOSO}_{4}$ solution, the milky white was disappeared. The aging time can reduce the rate of hydrolysis, influence the hydrolysis process and particle size and its distribution for metatitanic acid, eventually determine the performances of titanium dioxide pigment. With too long aging time, the nuclei activity would be reduces, particle aggregation would be serious, and pigment performance would be reduced. Suitable aging time was convenient to produce suitable nuclei and control the hydrolysis rate, resulting in better pigment properties. As for the low concentration $\mathrm{TiOSO}_{4}$ solution investigated, the aging time should be selected at $25 \mathrm{~min}$.

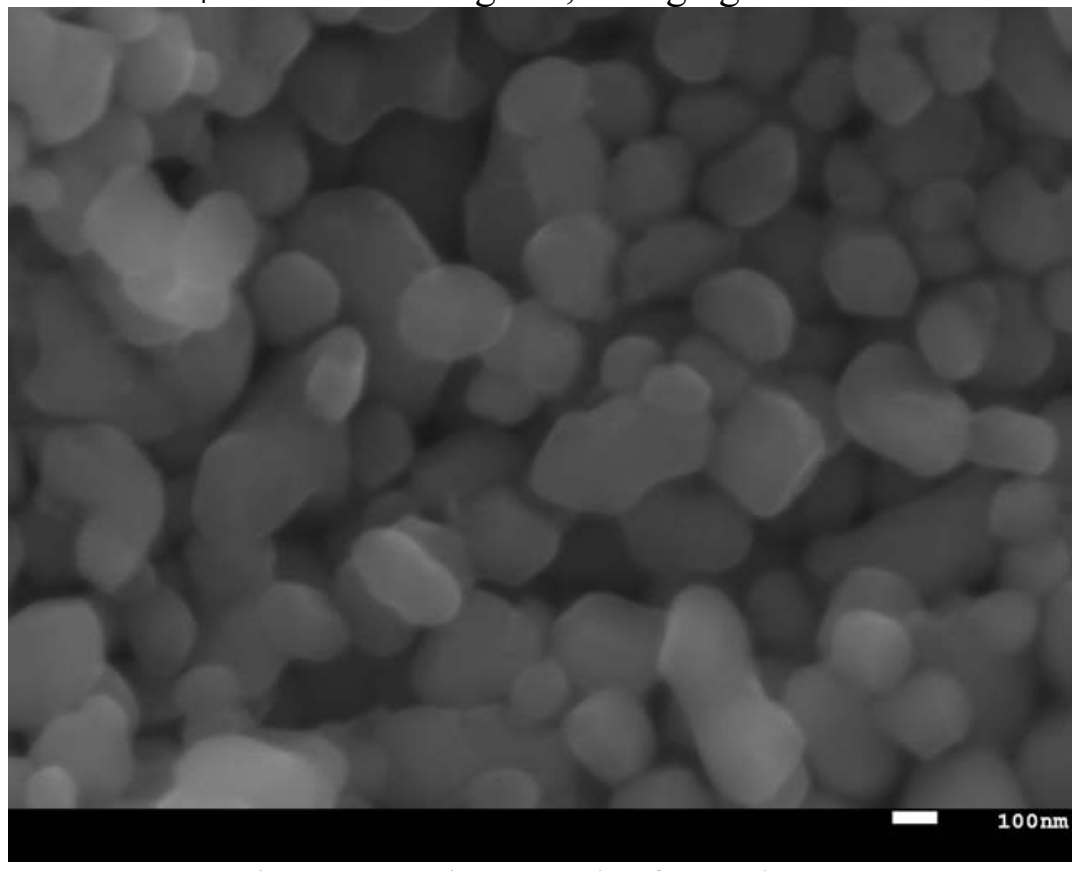

Fig.3 SEM photograph of sample R3 
The $\mathrm{F}$ value of the low concentration titanyl sulfate solution was adjusted by adding aqueous $\mathrm{NaOH}$ or $\mathrm{H}_{2} \mathrm{SO}_{4}$ solution, the hydrolysis of different $\mathrm{F}$ value of low concentration $\mathrm{TiOSO}_{4}$ solution was conducted at 1.60, 1.70, 1.80, 1.90, 2.00, and the prepared rutile titania samples were denoted as F1, F2, F3, F4, F5, respectively. The average particle size $\left(D_{A V}\right)$, particle polydispersity (Pdi) and pigment properties of the as-prepared rutile titania white pigments were listed in Table 2.

Table 2 Particle size distribution and pigment properties of titania obtained for different $\mathrm{F}$ value

\begin{tabular}{cccccccc}
\hline \multirow{2}{*}{ sample } & F value & $D_{A V} \mathrm{~nm}$ & Pdi & \multicolumn{2}{c}{ Achromatic ability } & \multicolumn{2}{c}{ Brightness } \\
\cline { 5 - 8 } & & & & TCS & SCX & Jasn $^{*}$ & Jasn $^{*} /$ Jasn $_{S} \%$ \\
\hline F1 & 1.60 & 336 & 0.317 & 1530 & 2.14 & 94.01 & 99.46 \\
F2 & 1.70 & 321 & 0.284 & 1610 & 2.73 & 94.35 & 99.82 \\
F3 & 1.80 & 311 & 0.205 & 1700 & 2.97 & 94.76 & 100.25 \\
F4 & 1.90 & 295 & 0.107 & 1790 & 3.22 & 95.10 & 100.61 \\
F5 & 2.00 & 281 & 0.279 & 1630 & 2.68 & 94.42 & 99.89 \\
R930 & $/$ & $\mathbf{2 8 8}$ & $\mathbf{0 . 1 6 7}$ & $\mathbf{1 9 1 0}$ & $\mathbf{3 . 1 2}$ & $\mathbf{9 4 . 5 2}$ & $/$ \\
\hline
\end{tabular}

The $\mathrm{F}$ value of the low concentration $\mathrm{TiOSO}_{4}$ solution will influence the velocity of nucleation, crystallization and aggregation of the hydrolysis, eventually determine the hydrolysis rate, space structure and particle size distribution of the product. All the products were with suitable particle size and narrow distribution, with sample F4 the best. The average particle size of $\mathrm{TiO}_{2}$ gradually decreased as F value increasing, and the Pdi value decreased first and then increased to 0.279 , with sample sample F4 the smallest, with the value of 0.107 . The pigment properties determination results showed sample F4 was the best, with reducing power (TCS) of 1790, blue phase index (SCX) of 3.22, Jasn of 95.10 , and the relative whiteness of $100.61 \%$ as to standard rutile sample R930. All the reducing power of the samples was lower than R930, and while blue phase index was first gradually increasing due to its forming smaller microcrystal and then decreasing owing to high temperature sintering for its wide particle size distribution. Higher F value inhibited the hydrolysis rate, decreased the number of hydrolysis nuclei initially formed, increasing the second nucleation, resulting in broad particle size distribution, eventually decreasing its flashback performance and pigment properties. Particle size distribution test and lower SCX value justified the discussion mentioned above. This also explained that proper number and quality of hydrolysis nuclei improved titanium white structure and its pigment properties. As for the low concentration $\mathrm{TiOSO}_{4}$ solution discussed, the optimized F value was of 1.90.

\section{Conclusions}

Aging time and influenced the crystal formation, nucleation, crystallization and aggregation process, eventually determined the crystal structure, particle size and its distribution of $\mathrm{TiO}_{2}$. All the as-prepared $\mathrm{TiO}_{2}$ samples were with appropriate particle size, narrow particle size distribution and good pigment properties. The optimized aging time after turning-grey-color point was of $25 \mathrm{~min}$, and the optimized $\mathrm{F}$ value was of 1.90 .

\section{Acknowledgments}

This work has been supported by the National Natural Science Foundation of China (50804025), Science and Technology Project of Sichuan Province, China (2014JY0197, 2011GZ0303), Project of Sichuan Provincial Human Resources and Social Security Department, Science and Technology Project of Panzhihua, China (2013CY-G-8, 2014CY-G-33, Guiding Project). 


\section{References}

[1] Tioxide Group Limited: Manufacture and General Properties of Titanium Dioxide Pigments, London, 1992.

[2] S. Karvinen, U.S. Patent 5,443,811. (1995)

[3] A. Przepiera, J. Sosnowski: Przem. Chem. Vol. 77 (1998), p. 328

[4] Ullmann's Encyclopedia of Industrial Chemistry. Weinheim: Wiley-VCH Verlag GmbH. (2002)

[5] Z. L. Tang: Production and environmental treatment of titanium dioxide. Peking: Chem. Ind. Press (2000)

[6] J. Z. Zhao, Z. C. Wang, L.W. Wang: Mater. Chem. Phy. Vol. 63(2000), p. 9

[7] S. Sathyamoorthy, M. J. Hounslob, G. D.Moggridge: Journal of Cryst. Growth Vol. 223 (2001), p. 225

[8] B. U.Grzmil, D.Grela, B. Kic: Pol. J. Chem. Technol. Vol. 11 (2009), p. 15

[9] I. Szilagyi, E. Konigsberger, P. M. May: Inorg. Chem. Vol. 48 (2009), p. 2200

[10] B. U. Grzmil, D. Grela, B. Kic: Chemical Pap. Vol. 63(2009), p. 217

[11] S. Sathyamoorthy, G. D. Moggridge, M. J. Hounslow: Cryst. Growth Des. Vol. 1 (2001), p.123 